\title{
Hernia due to iliac crest bone harvesting: A case report
}

\author{
Bisgin $\mathrm{T}^{*}$ and Sokmen $\mathrm{S}$ \\ Dokuz Eylul University Medical Faculty, Department of Surgery, İzmir, Turkey
}

\begin{abstract}
The most common source for the usage of autologous bone graft is the iliac crest in ortopedic reconstruction. Many complications after iliac crest bone harvesting have been reported. A rare and important complication of this procedure is the development of lumbar herniation. We herein report a case of right large lumbar hernia repaired with an open 'sandwich' repair technique, using both an expanded polytetrafluorene ethylene (Mycromesh $®)$ and a prosthetic polypropylene mesh.
\end{abstract}

\section{Introduction}

Lumbar hernias are quite rare with about 300 cases identified in the literature and only 20 of these are acquired traumatic hernia secondary to iliac crest bone graft harvesting [1]. Lumbar hernias occur within the superior and inferior lumbar triangle. Both of the triangles are regions of relatively weakness in the postero-lateral abdominal wall. Traumatic lumbar hernias located on the inferior lumbar triangle (Petit's triangle) are usually associated with iliac bone graft donor site. The diagnosis of this rare entity of abdominal hernia needs a high index of clinical suspicion. Sound surgical repair must be made against the possibility of incarceration (25\%) and strangulation (10\%) [2].

\section{Case presentation}

A 40-years-old woman with hypertension was presented with a mass in the right flank region, which had been gradually growing lately. She had previously undergone a right iliac crest bone harvest for cervical fusion operation. The mass which was easily reduced in the physical examination caused pain without creating symptoms of obstruction. The increased bowel sounds were heard in the auscultation. The patient underwent an elective open surgical intervention. A hernia originating from the inferior right lumbar triangle was revealed through the incision made over a large hernia sac in the right flank region. A $10 \times 10 \mathrm{~cm}$ created-defect extending from the right iliac crest to the subcostal region superiorly and from the lateral border of the anterior rectus sheath to the paraspinal muscles posteriorly was noted. Then, the defect was repaired using a $15 \times 15 \mathrm{~cm}$ expanded polytetrafluorene ethylene (e-PTFE) ( Mycromesh ${ }^{\circledR}$; W.L. Gore and Associates, Inc., Flagstaff, AZ) mesh subsequently, the musculoaponeurotic tissue was re-approximated over the mesh. Finally, a $15 \times 15 \mathrm{~cm}$ polypropylene mesh (Prolene ${ }^{\circledR}$; Ethicon, Somerville, NJ) was used to reinforce the musculoaponeurotic tissue (Figure 1a-d). There were no postoperative complications. The patient was discharged home after five days. At 2 years of follow up, she did not have any evidence of recurrence or any other complaint.

\section{Discussion}

Hernia development after iliac bone harvesting was first described by Oldfield in 1945 [3]. The rate of lumbar hernia development after iliac crest bone harvesting is 5-9\% [4]. A hernia can occur between 24 days and 15 years after a bone harvest. Symptoms occurred after 2 years

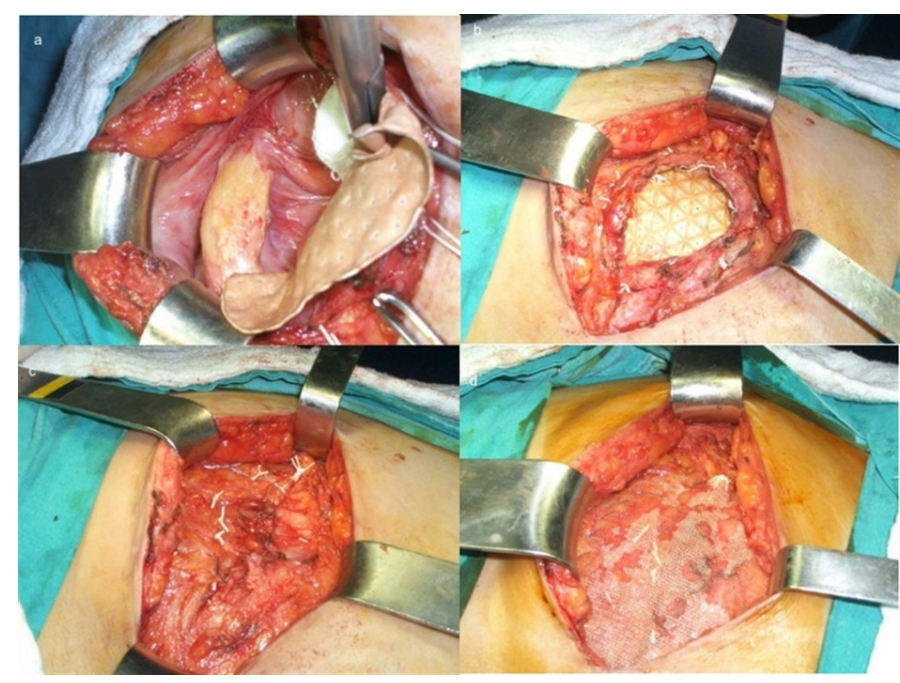

Figure 1. The defect was repaired using an e-PTFE mesh measuring approximately $15 \times 15$ $\mathrm{cm}$ subsequently, the musculoaponeurotic tissue was re-approximated over the mesh (Figure 1a-c), a $15 \times 15 \mathrm{~cm}$ polyproylene mesh was then placed on the musculoaponeurotic tissue (Figure 1d).

postoperatively in our patient. Certain risk factors are defined such as age, female gender, high BMI, weak abdominal muscles, a full thickness bone graft harvest, a deficit of more than $4 \mathrm{~cm}^{2}$, and chronic obstructive pulmonary disease [5].

The first symptom is an easily reducible uncomfortable, nontumoral mass of soft tissue in the incision area. If there is strangulation or incarceration, symptoms of intestinal obstruction can occur. Our patient was presented with a painful mass originating from the right flank region.

Various types of techniques have been used in the repair of traumatic lumbar hernia that developed after iliac crest bone harvesting. Bosworth's technique involved transferring the anterior

${ }^{\star}$ Correspondence to: Bisgin T, Dokuz Eylul University Medical Faculty, Department of Surgery, Izmir, Turkey, Tel: +905059062124, +902324122901; E-mail: tayfun.bisgin@gmail.com, tayfun.bisgin@deu.edu.tr

Received: July 02, 2018; Accepted: July 16, 2018; Published: July 20, 2018 
superior iliac spine below and drawing the muscle and fascia over the defect in the posterior [3]. The Dowd technique was described as the use of a pedicled gluteal fascial flap to cover the defect. In the Koontz technique, a lumbar fascial flap was used. In the Alexandre technique, a prosthetic mesh was fastened to the muscles and periosteum around the hernia [1]. A suture bone anchor or a corkscrew anchor can be used for fastening the mesh to the iliac crest [5]. Laparoscopic repair techniques have also been used with success. Very recently, good results have also been obtained with single incision endoscopic surgery [6].

We used a secure fixation technique which can be described as a modified Alexandre method to reconstruct the musculofascial defect as a complication of iliac crest bone harvesting. We ensured a durable repair by using both an e-PTFE and a polypropylene mesh on the patient. In cases where there is a large musculofascial defect during lumbar hernia repair, an e-PTFE mesh can be safely used for bridging the tissue loss and reinforcing it with a polypropylene mesh can provide a solid repair. One of the most crucial point in lumbar hernia repair is the stage of secure fixation of the mesh to the iliac crest, in which the recurrences most commonly occur. We successfully applicated the mycromesh to the iliac crest periosteum using CV-0 e-PTFE sutures (W.L. Gore and Associates, Inc., Flagstaff, AZ). No symptoms of recurrence or discomfort were seen after 2 years of patient follow-up.

\section{Conclusion}

Repairing a large lumbar hernia by using a double-layer ('sandwich') synthetic biomaterial is a sound and effective technique to reconstruct the abdominal wall loss due to iliac crest bone grafting.

\section{Informed consent}

Written informed consent was obtained from patient who participated in this staudy.

\section{Conflict of interest}

No conflict of interest was declared by the authors.

\section{Financial disclosure}

The authors declared that this study has received no financial support.

\section{References}

1. Yurcisin BM, Myers CJ, Stahfeld KR, Means JR (2010) Laparoscopic hernia repair following iliac crest harvest. Hernia 14: 93-96. [Crossref]

2. Radais F, Facy O, Ortega-Deballon P (2011) Transiliac hernia. Am J Surg 201: e41-42. [Crossref]

3. BOSWORTH DM (1955) Repair of herniae through iliac-crest defects. J Bone Joint Surg Am 37-37A: 1069-73. [Crossref]

4. Forrest C, Boyd B, Manktelow R, Zuker R, Boven V (1992) The free vascularised iliac crest tissue transfer: donor site complication associated with eighty-two cases. $\mathrm{Br} J$ Plast Surg 45: 89-93. [Crossref]

5. Fontaine V, Villeneuve PL, Belzile EL, Laflamme M (2016) Hernia through an iliac crest bone graft harvest site: two cases treated differently. J Clin Case Rep 6: 798.

6. Kawaguchi M, Ishikawa N, Shimizu S, Shin H, Matsunoki A, et al. (2011) Single incision endoscopic surgery for lumbar hernia. Minim Invasive Ther Allied Technol 20: $62-64$.

Copyright: (C2018 Bisgin T. This is an open-access article distributed under the terms of the Creative Commons Attribution License, which permits unrestricted use, distribution, and reproduction in any medium, provided the original author and source are credited. 